\title{
Rehabilitation Training and Resveratrol Improve the Recovery of Neurological and Motor Function in Rats after Cerebral Ischemic Injury through the Sirt1 Signaling Pathway
}

\author{
Na Shi, ${ }^{1}$ Chongtian Zhu, ${ }^{1}$ and Liying $\mathrm{Li}^{2}$ \\ ${ }^{1}$ Rehabilitation Department, Linyi People's Hospital, Linyi, China \\ ${ }^{2}$ Department of Rehabilitation, Shandong Medical College, Jinan, China \\ Correspondence should be addressed to Liying Li; liliying2022@126.com
}

Received 3 August 2016; Revised 15 October 2016; Accepted 6 November 2016

Academic Editor: Leon Spicer

Copyright (C) $2016 \mathrm{Na}$ Shi et al. This is an open access article distributed under the Creative Commons Attribution License, which permits unrestricted use, distribution, and reproduction in any medium, provided the original work is properly cited.

\begin{abstract}
This study was conducted to investigate the recovery of motor function in rats through the silent information regulator factor 2-related enzyme 1 (Sirtl) signal pathway-mediated rehabilitation training. Middle cerebral artery occlusion (MACO) was used to induce ischemia/reperfusion injury. The rats were subjected to no treatment (model), rehabilitation training (for 21 days), resveratrol ( $5 \mathrm{mg} / \mathrm{kg}$ for 21 days), and rehabilitation training plus resveratrol treatment. $24 \mathrm{~h}$ later, They were assessed for neurobehavioral score and motor behavior score and expression of brain derived-nerve neurotrophic factor (BDNF) and tyrosine kinase receptor B (TrkB). Compared with sham group, models had significantly higher neurobehavioral scores, balance beam, and rotary stick scores. Compared with the model group, rats in rehabilitation training and resveratrol groups had significantly reduced scores. Compared with rehabilitation training or resveratrol treatment alone, rehabilitation plus resveratrol further reduced the scores significantly. The percentage of cells expressing BDNF and TrkB and expression levels of BDNF and TrkB were similar between the model and sham groups, significantly increased in rehabilitation training and resveratrol groups, and further increased in rehabilitation training plus resveratrol group. These results indicate that rehabilitation raining plus resveratrol can significantly improve the recovery of motor function in rats after cerebral ischemic injury, which is likely related to the upregulation of the BDNF/TrkB signaling pathway.
\end{abstract}

\section{Introduction}

Cerebrovascular disease is a major hazard to human health and life. Ischemia resulting from middle cerebral artery occlusion (MCAO) accounts for nearly $80 \%$ of cerebrovascular diseases, which have higher incidence, disability, and mortality rate and are heavy burden to the patient's family and society [1-3]. At present, the clinical treatments of ischemic cerebral vascular diseases is mainly relied on early thrombolysis, nerve protection and rehabilitation. Among them, rehabilitation training is most widely used, which helps to improve the patient's body movement, feeling, language, and cognition ability. Studies have shown that rehabilitation training can increase cerebral blood flow, promote the survival of neurons after cerebral infarction, inhibit cerebral swelling, and stimulate the secretion of neuron growth factors and neurotrophic factors to improve or restore nerve and limb movement ability [4-7]. However, in most of the previous studies, drug treatment or rehabilitation training alone is used to improve the neurological and motor function. Fewer studies have dealt with combined therapy of drug and rehabilitation.

Silent information regulator factor 2-related enzyme 1 (Sirtl) is a member of the sirtuins family. It is an acetylated protein closely related to the age and aging. Studies have shown that Sirtl is not only associated with inflammation, osteoarthritis, diabetes, cardiovascular disease, and cancer, but also associated with the progression of neurodegenerative diseases [8-10]. A large number of studies have shown that Sirtl or its agonist resveratrol has a significant protective effect 
on neurons in rats after middle cerebral artery occlusion (MCAO) [11-15]. In addition, antioxidant transcription factor Nrf2 is also activated by resveratrol to upregulate the target genes such as $\mathrm{NAD}(\mathrm{P}) \mathrm{H}$ :quinone oxidoreductase 1 , $\gamma$-glutamylcysteine synthetase, and heme oxygenase- 1 to protect endothelial cells [16]. However, other mechanisms underlying resveratrol mediated protection, particularly when used in combination with physical therapy, need to be further explored.

In this study, we investigate the effect of combined therapy of rehabilitation training plus resveratrol on the recovery of neural function, motor function in MCAO rats, and possible mechanisms underlying the effect. The findings would provide insights into additional role of resveratrol and rehabilitation for better clinical therapeutic use.

\section{Materials and Methods}

2.1. Animals. Two-month-old male SD rats (SPD grade), weighting $200 \pm 20 \mathrm{~g}$, were purchased from Silaike Experimental Animal Co., Ltd., Shanghai (certificate number 20120002) and were hosted in animal rooms at $23 \pm 2^{\circ} \mathrm{C}$ with free access to food and drinking water.

2.2. Reagents and Instruments. Resveratrol was purchased from Sigma (lot number 34092-100 mg), USA; BCA protein assay kit, mouse anti-GAPDH monoclonal antibody, and horseradish peroxidase-labeled goat anti-rabbit IgG $(\mathrm{H}$ plus L) were purchased from Beytime Biotechnology (lots numbers P0010, AG019, and A0208), Beijing; rabbit antiBDNF and TrkB polyclonal antibodies were purchased from Abcam (lots numbers ab108319 and ab18987), USA; Trizol kit and One-Step RT-PCR kit were purchased from Invitrogen (lots numbers 15596-018 and AM1005), USA. Electrophoresis apparatus, transfer apparatus, and gel imaging system were purchased from Bio-Rad, USA; plate reader was from TECAN, Swiss, and fluorescence microscope AF6000 was obtained from Leica, Germany. All animal experimental protocols were approved by the research ethic committee of Linyi Chinese Medicine Hospital.

2.3. Modeling. MCAO models were constructed as previously described $[4,5]$. Briefly, the rats were anaesthetized using $10 \%$ chloric aldehyde. The right common carotid artery (CCA), right internal carotid artery (ICA), and right external carotid artery (ECA) were separated, ECA and ICA were ligated, and ICA was clipped. A small incision was made on ECA near CCA and inserted slowly with a nylon line to a depth of about $18 \mathrm{~mm}$ to generate MCAO. After $2 \mathrm{~h}$ of ischemia, the nylon line was slightly withdraw to allow reperfusion for $22 \mathrm{~h}$. For sham group, ECA, CCA, and ICA were separated but not ligated.

2.4. Grouping and Drug Treatments. Rats were equally grouped into sham $(n=24)$, model $(n=24)$, rehabilitation training $(n=24)$, resveratrol $(n=24)$, and rehabilitation plus resveratrol groups $(n=24)$. Rehabilitation training started $24 \mathrm{~h}$ after the operation as described $([4,5])$, including crawling on balance beams and rotary bars rotating alternatively clockwise and counterclockwise at $3 \mathrm{rpm}$. The training lasted 10 min twice daily for 21 days. Resveratrol was given by daily abdominal injection with $5 \mathrm{mg} / \mathrm{kg}$ resveratrol for 21 days [17].

2.5. Neurological Behavior and Motor Function Assessment. Twenty-four hours after the surgery, the rats were assessed for neurological function based on the Longa Zea scoring method [4,5]. Rats scored 1 to 3 were randomized for subsequent experiments. They were assessed again at 2, 7, 14, and 21 days after surgery before rehabilitation training and resveratrol administration. The rats were also assessed for motor function before rehabilitation training and resveratrol administration as previously described [6].

2.6. Tissue Sampling. 21 days after the surgery, rats were sacrificed and the brain tissues were isolated and washed in prechilled saline. Cortex tissues taken from middle cerebral artery area were fixed in $10 \%$ neutral formalin for immunohistochemical assay or stored at $-80^{\circ} \mathrm{C}$ for Western blot and RT-PCR analyses.

2.7. Immunohistochemical Assay. Fixed cortex tissues were imbedded in paraffin and sectioned and immersed in 3\% hydrogen peroxide for $15 \mathrm{~min}$ to remove endogenous peroxidase. After being blocked with nonimmune animal serum and washed with PBS, the slides were incubated in primary antibody solutions at room temperature for $2 \mathrm{~h}$, washed with PBS, and incubated with horseradish peroxidase-labeled goat anti rabbit $\operatorname{IgG}(\mathrm{H}+\mathrm{L})$ at $37^{\circ} \mathrm{C}$. The slides were developed in DAB solution and counterstained with hematoxylin. Dark brown or yellow-browed colored cells were considered positive, and the positive percentages were calculated using Image J software based on average light density.

2.8. RT-PCR. BDNF and TrkB mRNA expression was analyzed by RT-qPCR on total RNA isolated from cortex tissues using the Trizol reagent according to the supplier's protocols. Reverse transcription was performed with $200 \mathrm{ng}$ of RNA in a total volume of $10 \mu \mathrm{L}$ using One-Step RTPCR kit according to manufacturer's recommendations. The amplified products were separated on $2 \%$ agarose gel by electrophoresis. RT-qPCR was performed on the 7900HT Fast Real-Time PCR system using TaqMan gene expression assays probes (Applied Biosystems). The primers used for BDNF were 5'-TATCCTTATGAACCGCCAGCC (forward) and $5^{\prime}$-TTCTGACATTAAGGGCCGTG (reverse) and for TrkB were $5^{\prime}$-CAAGTTGGCGAGACATTCCA (forward) and $5^{\prime}$-AGTCATCGTCGTTGCTGATGAC (reverse). Human glyceraldehyde-3-phosphate dehydrogenase, GADPH (Hs03929097_g1), was amplified using a pair of primers $5^{\prime}$-AGCCACATCGCTCAGACA (forward) and $5^{\prime}$-TGGACTCCACGACGTACT (reverse) as an internal control. The PCR was carried out in a total volume of $10 \mu \mathrm{L}$ containing $1.5 \mu \mathrm{L}$ of diluted and preamplified cDNA, $10 \mu \mathrm{L}$ of TaqMan Gene Expression Master Mix, and $1 \mu \mathrm{L}$ of each fluorescence TaqMan probe. The cycling conditions were $50^{\circ} \mathrm{C}$ for $2 \mathrm{~min}, 95^{\circ} \mathrm{C}$ for $10 \mathrm{~min}$ followed by 40 cycles, each 
TABLE 1: Neurobehavioral scores of rats following middle cerebral artery occlusion/reperfusion injury, rehabilitation training, and resveratrol treatment.

\begin{tabular}{|c|c|c|c|c|}
\hline \multirow{2}{*}{ Groups } & \multicolumn{4}{|c|}{ Days after the surgery } \\
\hline & 2 & 7 & 14 & 21 \\
\hline Sham & $0^{\mathrm{A}}$ & $0^{\mathrm{A}}$ & $0^{\mathrm{A}}$ & $0^{\mathrm{A}}$ \\
\hline Model & $2.38 \pm 0.24^{* *, \mathrm{~A}}$ & $1.79 \pm 0.18^{* *, \mathrm{~B}}$ & $1.37 \pm 0.13^{* *, \mathrm{~B}}$ & $1.00 \pm 0.10^{* *, \mathrm{C}}$ \\
\hline Rehabilitation training & $2.01 \pm 0.15^{* *, A}$ & $0.88 \pm 0.09^{* *, \# \#, \mathrm{~B}}$ & $0.59 \pm 0.05^{* *, \# \#, \mathrm{C}}$ & $0.28 \pm 0.03^{\# \#, \mathrm{D}}$ \\
\hline Resveratrol & $2.00 \pm 0.12^{* *, \mathrm{~A}}$ & $0.87^{* *,} \pm 0.08^{* *, \#, \mathrm{~B}}$ & $0.60 \pm 0.06^{* *, \# \#, \mathrm{C}}$ & $0.29 \pm 0.03^{\# \#, \mathrm{D}}$ \\
\hline Rehabilitation training plus resveratrol & $1.96 \pm 0.17^{* *, \# \#, \mathrm{~A}}$ & $0.56^{* *,} \pm 0.05^{\# \#,++, \mathrm{B}}$ & $0.40 \pm 0.04^{\# \#,++, \mathrm{C}}$ & $0.12 \pm 0.01^{\# \#,++, \mathrm{D}}$ \\
\hline
\end{tabular}

**,\#\#, and ++ denote values with $P<0.01$ versus sham, model, and rehabilitation training or resveratrol, respectively. Numbers labelled with different capital letters in the same row are significantly different $(P<0.01)$ based on repeated measures ANOVA.

one consisting of $45 \mathrm{~s}$ at $95^{\circ} \mathrm{C}$ and $45 \mathrm{~s}$ at $59^{\circ} \mathrm{C}$ with final extension at $72^{\circ} \mathrm{C}$ for $60 \mathrm{~s}$. Samples were run in triplicate and the mean value was calculated for each case.

The data were managed using the Applied Biosystems software RQ Manager v1.2.1. Relative expression was calculated by using comparative $\mathrm{Ct}$ method and obtaining the fold change value $\left(2^{-\Delta \Delta \mathrm{Ct}}\right)$ according to previously described protocol [18].

2.9. Western Blot. To detect BDNF and TrkB protein expression, total proteins were extracted from the brain tissue in RIPA lysis buffer. The protein concentration was determined using the BCA kit. Sixty $\mu \mathrm{g}$ of the proteins was separated by SDS-PAGE and transferred (Bio-Rad, USA) to PVDF membranes (Millipore, USA). BDNF and TrkB protein expression levels were quantified using rabbit polyclonal antibodies specific for each protein. The expression levels of these proteins were standardized to human GADPH using a mouse polyclonal anti-GADPH antibody. Primary antibodies were detected using goat anti-rabbit or goat anti-mouse horseradish peroxidase- (HRP-) conjugated secondary antibodies. Immunoreactive bands were visualized using Western Lighting Chemiluminescence Reagent Plus (PerkinElmer, USA) according to the manufacturer's instructions and then quantified by densitometry using a Bio-Rad gel imaging system and Quantity One software.

2.10. Statistical Analysis. All data were expressed as means \pm standard error of the mean (SEM) obtained from at least three independent experiments and analyzed using the statistical software SPSS 17. Means were compared using oneway ANOVA or repeated measures ANOVA. Tukey's test was used as the post hoc test to determine mean differences within groups. A $P$ value $\leq 0.05$ was considered statistically significant.

\section{Results}

3.1. Neurobehavioral Assessment. As shown in Table 1, the neurobehavioral scores were significantly higher in the model group than in the sham groups 7 days after operation $(P<$ $0.01)$, while the rats in rehabilitation training and resveratrol groups had significantly lower scores as compared to those in the model group $(P<0.01)$. Furthermore, the scores were further lowered in rehabilitation training plus resveratrol group $(P<0.01)$. These score decreases were statistically significant within 7 days of treatment and more remarkable as the time increased after the surgery (Table 1). In most cases, there were significantly effects of time on the outcome of neurobehavioral scores except for sham (Table 1). ANOVA analyses showed that rehabilitation training, resveratrol, rehabilitation training plus resveratrol, and therapy time had significantly impact on improvement of neurobehavioral scores $(F=5.37,4.22,4.03$, and 9.12, resp., $P<0.01)$.

3.2. Kinematic Behavior Assessment. We then assessed the kinematics behavior scores of the rats and the results are shown in Tables 2 and 3. Seven days after operation, balance beam and rotary stick scores were significantly $(P<0.01)$ higher in the model groups than in the sham group and significantly $(P<0.01)$ lower in rehabilitation training group and resveratrol group than in model group. The scores were further reduced significantly $(P<0.01)$ in rehabilitation plus resveratrol group). These scores began to decrease significantly after 7 days of treatment throughout entire experimental period (Tables 2 and 3). In most cases, there were significant effects of time on the outcome of balance beam scores and rotary stick scores except for sham (Tables 2 and 3). In ANOVA analysis rehabilitation training, resveratrol, rehabilitation training plus resveratrol, and therapy time had shown significant variation in balance beam scores $(F=5.57,6.22,3.03$, and 7.12, resp., $P<0.01)$ and rotary stick scores $(F=4.37,5.22,7.03$, and 5.12, resp., $P<0.01)$.

3.3. Expression of BDNF and TrkB. To analysis changes at the molecular level, we assessed the expression of BDNF and $\operatorname{TrkB}$ in the brain tissues 21 days after the operation and the results are shown in Table 4 and Figures 1-4. In comparison with sham group, the positive percentages, protein, and mRNA levels for the two genes did not change in the model group $(P>0.05)$. However, these parameters were significantly $(P<0.01)$ increased in rehabilitation training or resveratrol groups and were further increased significantly $(P<0.01)$ in rehabilitation training plus resveratrol group.

\section{Discussion}

Ischemic cerebrovascular disease or ischemic stroke is one of the major causes of disability in the elderly. The incidence of the disease is increasing rapidly as the population ages 
TABLE 2: Balance beam scores of rats following middle cerebral artery occlusion/reperfusion injury, rehabilitation training, and resveratrol treatment.

\begin{tabular}{|c|c|c|c|c|}
\hline \multirow{2}{*}{ Groups } & \multicolumn{4}{|c|}{ Days after the surgery } \\
\hline & 2 & 7 & 14 & 21 \\
\hline Sham & $1.00 \pm 0.00^{\mathrm{A}}$ & $1.00 \pm 0.00^{\mathrm{A}}$ & $1.00 \pm 0.00^{\mathrm{A}}$ & $1.00 \pm 0.00^{\mathrm{A}}$ \\
\hline Model & $5.08 \pm 0.52^{* *, \mathrm{~A}}$ & $2.79 \pm 0.28^{* *, \mathrm{~B}}$ & $2.24 \pm 0.3^{* *, \mathrm{~B}}$ & $1.69 \pm 0.17^{* *, \mathrm{C}}$ \\
\hline Rehabilitation training & $5.01 \pm 0.50^{* *, \mathrm{~A}}$ & $1.83 \pm 0.19^{* *, \# \#, \mathrm{~B}}$ & $1.48 \pm 0.15^{* *, \# \#, \mathrm{C}}$ & $1.19 \pm 0.12^{* *, \# \#, \mathrm{D}}$ \\
\hline Resveratrol & $5.00 \pm 0.52^{* *, \mathrm{~A}}$ & $1.85 \pm 0.18^{* *, \# \#, \mathrm{~B}}$ & $1.49 \pm 0.15^{* *, \# \#, \mathrm{~B}}$ & $1.20 \pm 0.12^{* *, \# \#, \mathrm{C}}$ \\
\hline Rehabilitation training plus resveratrol & $4.98 \pm 0.17^{* *, \mathrm{~A}}$ & $1.45 \pm 0.15^{* *, \# \#,++, \mathrm{B}}$ & $1.23 \pm 0.12^{* *, \# \#,++, C}$ & $1.06 \pm 0.10^{\# \#,++, \mathrm{D}}$ \\
\hline
\end{tabular}

$* *$,\#, and ++ denote values with $P<0.01$ versus sham, model, and rehabilitation training or resveratrol, respectively. Numbers labelled with different capital letters in the same row are significantly different $(P<0.01)$ based on repeated measures ANOVA.

TABLE 3: Rotary stick scores of rats following middle cerebral artery occlusion/reperfusion injury, rehabilitation training, and resveratrol treatment.

\begin{tabular}{lcccc}
\hline Groups & & \multicolumn{3}{c}{ Days after the surgery } \\
& 2 & 7 & $0^{\mathrm{A}}$ & 21 \\
\hline Sham & $0^{\mathrm{A}}$ & $0^{\mathrm{A}}$ & $0^{\mathrm{A}}$ \\
Model & $2.28 \pm 0.22^{* *, \mathrm{~A}}$ & $1.95 \pm 0.19^{* *, \mathrm{~B}}$ & $1.46 \pm 0.13^{* *, \mathrm{C}}$ & $1.12 \pm 0.11^{* *, \mathrm{D}}$ \\
Rehabilitation training & $2.21 \pm 0.20^{* *, \mathrm{~A}}$ & $1.42 \pm 0.13^{* *, \# \#, \mathrm{~A}}$ & $1.07 \pm 0.11^{* *, \#, \mathrm{C}}$ & $0.53 \pm 0.05^{* *, \# \#, \mathrm{D}}$ \\
Resveratrol & $2.20 \pm 0.22^{* *, \mathrm{~A}}$ & $1.43 \pm 0.14^{* *, \# \#, \mathrm{~B}}$ & $1.05 \pm 0.10^{* *, \#, \mathrm{C}}$ & $0.53 \pm 0.05^{* *, \# \#, \mathrm{D}}$ \\
Rehabilitation training plus resveratrol & $2.17 \pm 0.21^{* *, \mathrm{~A}}$ & $1.10 \pm 0.10^{* *, \#,++, \mathrm{B}}$ & $0.90 \pm 0.09^{* *, \# \#,++, \mathrm{B}}$ & $0.36 \pm 0.04^{* *, \# \#,++, \mathrm{C}}$ \\
\hline
\end{tabular}

$* *$,\#, and ++ denote values with $P<0.01$ versus sham, model, and rehabilitation training or resveratrol, respectively. Numbers labelled with different capital letters in the same row are significantly different $(P<0.01)$ based on repeated measures ANOVA.

TABLE 4: Positive percentages of BDNF and TrkB in brain tissue of following middle cerebral artery occlusion/reperfusion injury, rehabilitation training, and resveratrol treatment.

\begin{tabular}{lcc}
\hline Groups & $\begin{array}{c}\text { Positive } \\
\text { percentage of } \\
\text { BDNF }\end{array}$ & $\begin{array}{c}\text { Positive } \\
\text { percentage of } \\
\text { TrkB }\end{array}$ \\
\hline Sham & $28.17 \pm 2.82$ & $18.90 \pm 1.89$ \\
Model & $29.86 \pm 2.99$ & $20.17 \pm 0.21$ \\
$\begin{array}{l}\text { Rehabilitation } \\
\text { training }\end{array}$ & $42.35 \pm 4.40^{\# \#}$ & $38.47 \pm 3.85^{\# \#}$ \\
$\begin{array}{l}\text { Resveratrol } \\
\text { Rehabilitation } \\
\text { training plus } \\
\text { resveratrol }\end{array}$ & $42.29 \pm 4.23^{\# \#}$ & $38.49 \pm 3.85^{\# \#}$ \\
\end{tabular}

$\# \#$ and ++ denote values with $P<0.01$ versus sham, model, and rehabilitation training or resveratrol, respectively.

and recovery of motor functions after ischemic stroke has attracted increasingly attentions [1-3]. While rehabilitation training has been shown to be effective for improving the conditions of stroke patients for motor function and cognitive ability [4-7], the molecular mechanisms are still poorly understood.

As a member of silence information regulator 2 (Sir2) family, Sirtl is a nicotinamide adenine dinucleotide- $\left(\mathrm{NAD}^{+}{ }_{-}\right)$ dependent histone deacetylase. It regulates the transcription of a number of downstream genes through $\mathrm{NAD}^{+}$to participate in various cellular processes such as apoptosis, cell cycle, cell energy metabolism, oxidative stress, and cell aging process and is involved in a number of diseases such as diabetes, tumor, inflammation, aging, and neural degenerative diseases [8-10]. Earlier studies have revealed that the upregulation of Sirtl activity or use of Sirtl agonist resveratrol attenuates neurological injury in rats after cerebral ischemia through increased antioxidant capacity and neuronal survival although the mechanisms are largely unknown [11-15]. Also, it is not clear if rehabilitation training and Sirtl agonist resveratrol have synergy in the recovery of motor functions after cerebral ischemia.

Our study shows that, from 7 to 21 days after operation, the recovery of motor functions in MACO rats was significant in a time-dependent manner as indicated by decreased neurobehavioral scores, balance beam, and rotary stick scores after rehabilitation training or resveratrol treatment. These results are consistent with early works $[6,19]$. Furthermore, these scores were further reduced in rats receiving rehabilitation training plus resveratrol, suggesting that there is synergy between rehabilitation training and resveratrol.

Research shows that the recovery of damaged nerve after cerebral ischemia may be promoted through the secretion of various neurotrophic factors, which bind to their receptors to activate downstream signaling systems [20, 21]. BDNF is a neurotrophic factor widely distributed in endocrine cells, bone tissues, the central nervous system, and the peripheral nervous system. It is shown to promote neuronal growth, survival, and injury repair and plays significant role in neurogenesis and neurodegenerative diseases [20, 21]. BDNF has two kinds of receptors. One is low affinity receptor p75, capable of binding with all the neurotrophic factor families, and the other is Trk, including TrkA, TrkB, and $\operatorname{TrkC}$. TrkB is known to be the strongest receptor of BDNF and is mostly studied. BDNF binds TrkB to form 


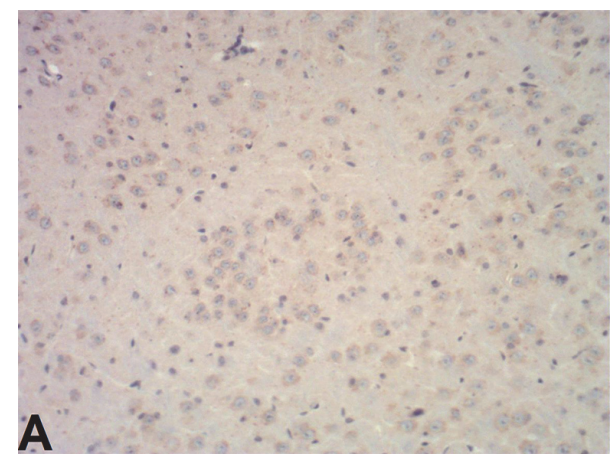

(a)

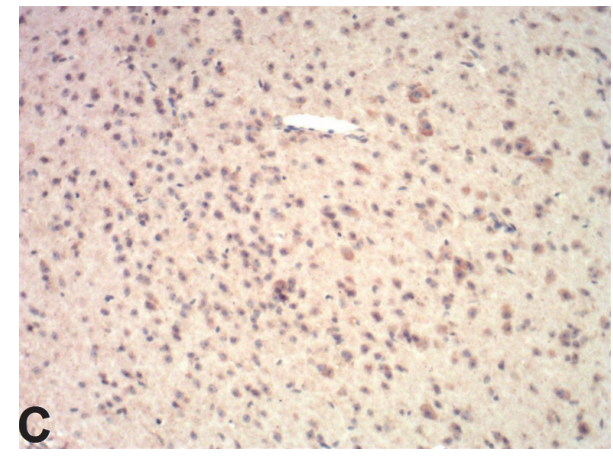

(c)

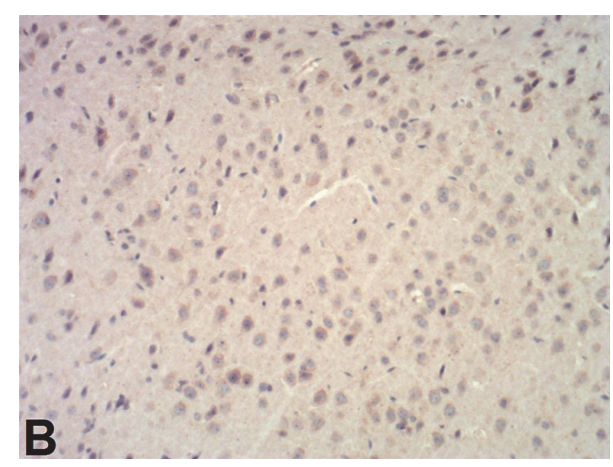

(b)

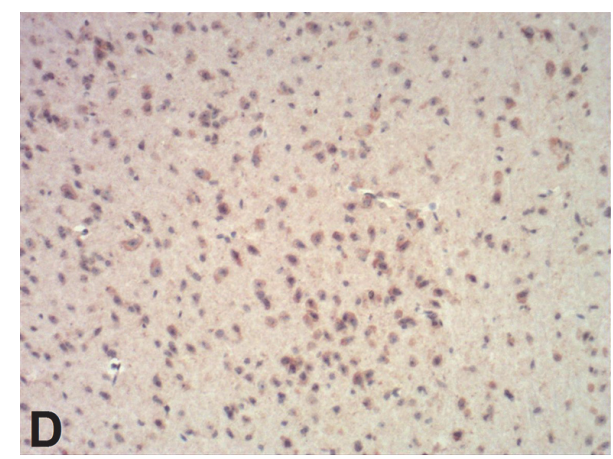

(d)

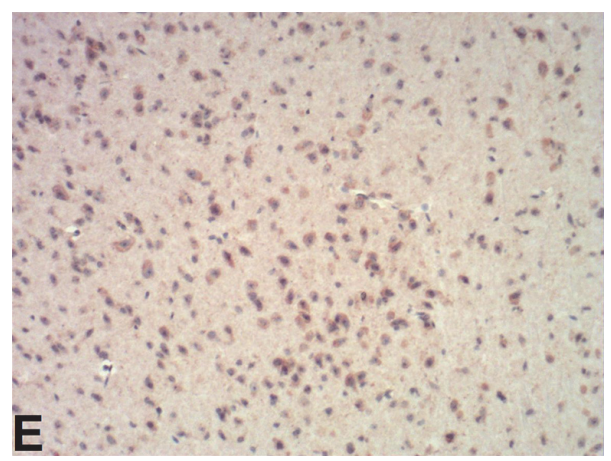

(e)

FIGURE 1: Immunohistochemistry staining of brain cells for BDNF $(\times 400)$. (a) Sham operation group; (b) model group; (c) rehabilitation training group; (d) resveratrol group; (e) rehabilitation training plus resveratrol group.

ligand-receptor complex to activate tyrosine kinase and then downstream signaling pathways such as phosphatidylinositol 3 kinase (PI3K), extracellular regulating kinase (ERK), and mitogen-activated protein kinase (MAPK) signaling pathway to regulate synaptic function and synthesis of neuronal proteins and restore neuronal regeneration and survival [22, 23]. Research has shown that in cerebral ischemic rats BDNF/ TrkB expression is upregulated to stabilize ischemia-induced $\mathrm{Ca}^{2+}$ imbalance, inhibit the toxic effect of excitatory amino acids on neurons, and stimulate the survival and regeneration of damaged neurons as well as differentiation and proliferation of neural stem cells [24-26]. Rehabilitation training has been found to increase the expression of BDNF in cerebral ischemia mice [27] and in the serum of patients with Parkinson's disease [28]. On the other hand, resveratrol is shown to increase the expression of Sirtl as well as BDNF and its receptor TrkB [29] in elderly rats, suggesting that Sirt1 might protect retinal neurons and visual function through the regulation of BDNF/TrkB. Sirtl is also shown to have neuroprotective effect in patients with Huntington's disease, and the effect is associated with the upregulation of BDNF/ TrkB signaling pathway [30]. These results imply that the recovery of neural function and motor function in rats with cerebral ischemia may be related to the BDNF/TrkB signaling pathway. To elucidate the molecular mechanisms of the functional recovery, we analyzed the expression of BDNF 


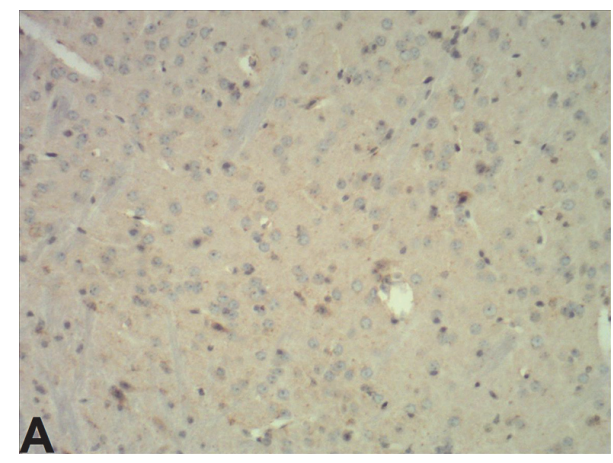

(a)

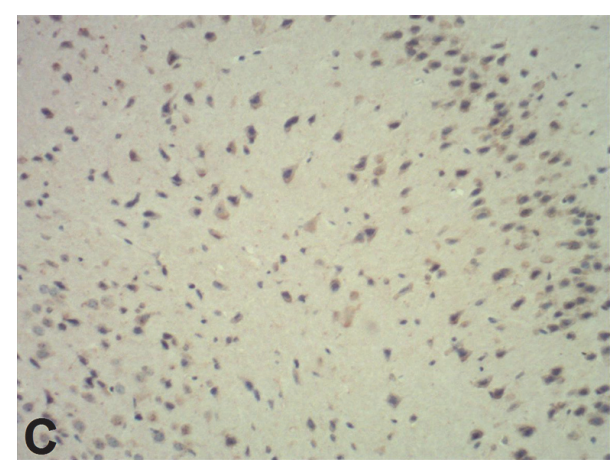

(c)

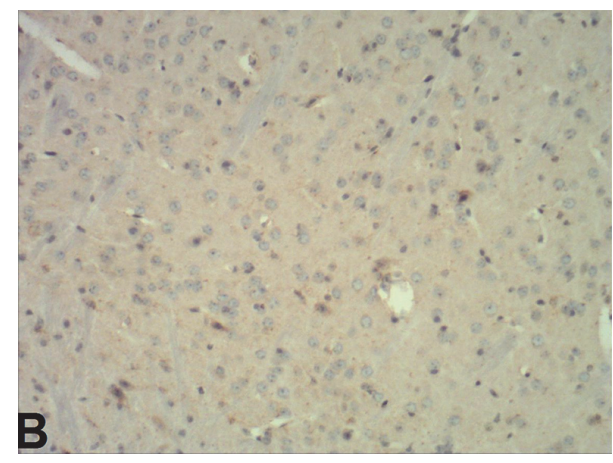

(b)

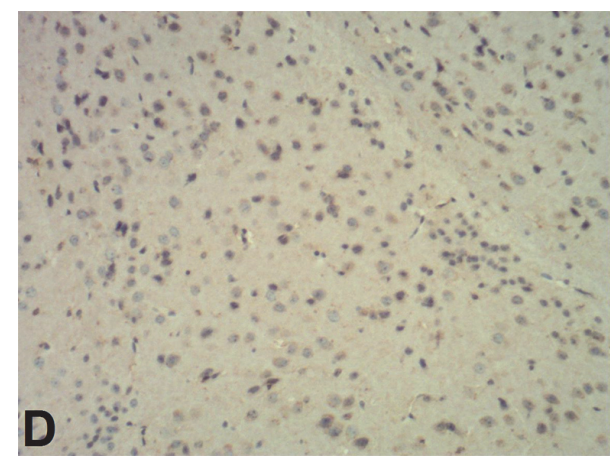

(d)

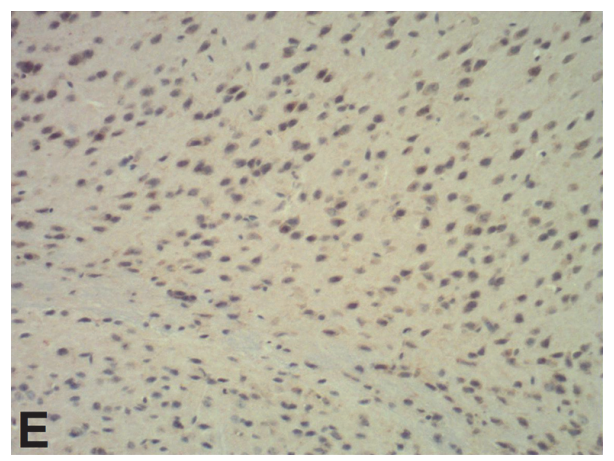

(e)

FIGURE 2: Immunohistochemistry staining of brain cells for $\operatorname{TrkB}(\times 400)$. (a) Sham operation group; (b) model group; (c) rehabilitation training group; (d) resveratrol group; (e) rehabilitation training plus resveratrol group.

and Trk in the brain tissues. Our study shows that 21 days after surgery, there was no difference in BDNF/TrkB expression at protein and mRNA levels between the sham and model groups, which is similar to the early study [31]. However, between 3 and 14 days after operation, the model group had higher but gradually decreasing BDNF/TrkB expression as compared to the rats in sham group. This might be due to the secretion of endogenous neurotrophic factors in early cerebral ischemia stage that restores the nerve and motor functions, while in late cerebral ischemia stage, the self-recovery is decreased, resulting in a decrease in expression of BDNF and TrkB. On the other side, the expression of BDNF and TrkB was significantly increased after rehabilitation training and resveratrol treatment, suggesting that both therapies may upregulate the BDNF/TrkB signaling pathway, resulting in the recovery of nervous system function and motor function in the cerebral ischemic rats. Resveratrol has been shown to activate antioxidant transcription factor Nrf2 to confer endothelial protection via upregulation of target genes that reduce the oxidative stress [16], a protective mechanism different from the one observed in our study. Furthermore, further increase in BDNF and TrkB expression in the combined rehabilitation training and resveratrol treatment as compared to single 


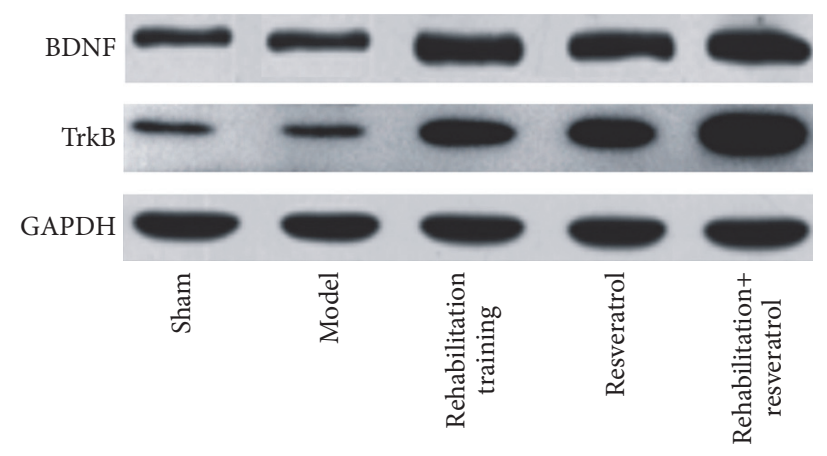

(a)

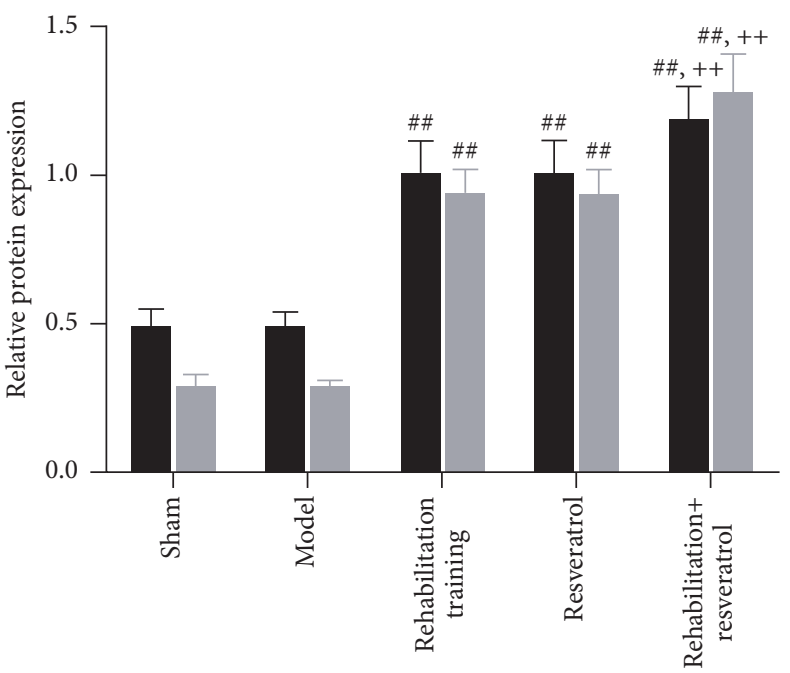

BDNF/GAPDH $\operatorname{TrkB} / \mathrm{GAPDH}$

FIGURE 3: BDNF and TrkB protein expression in brain tissues 21 days after the operation. (a) Representative Western blot; (b) relative protein content. \#\# and ++ denote values with $P<0.01$ versus sham, model, and rehabilitation training or resveratrol, respectively.

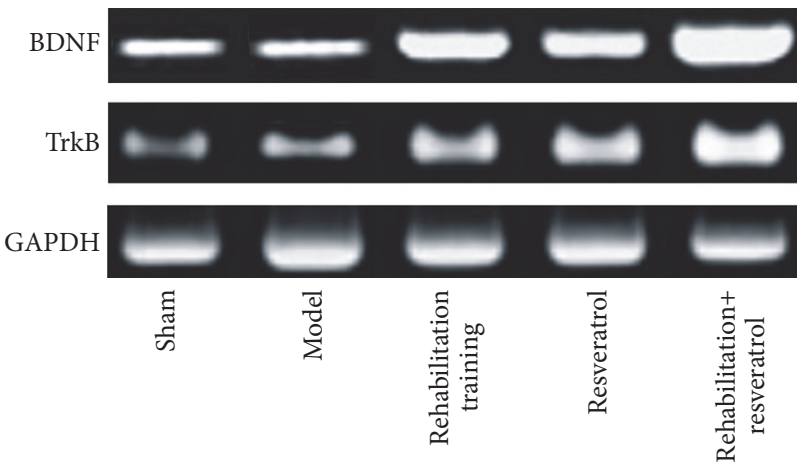

(a)

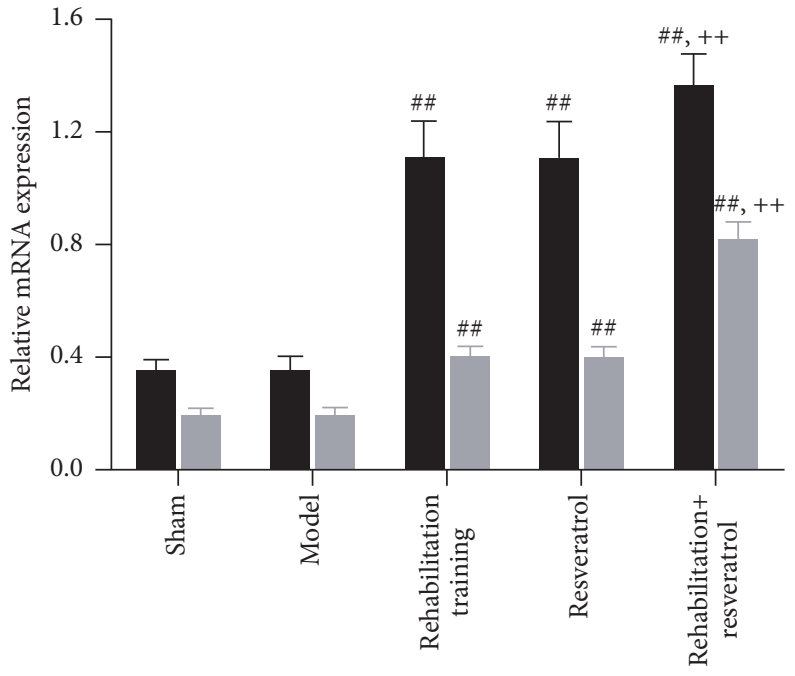

BDNF/GAPDH

TrkB/GAPDH

(b)

Figure 4: PCR analysis of BDNF and TrkB mRNA expression in brain tissues. (a) Electrophoresis of PCR product; (b) relative mRNA levels. $\# \#$ and ++ denote values with $P<0.01$ versus sham, model, and rehabilitation training or resveratrol, respectively.

treatment was observed, indicating that there is synergistic effect on the neurological function and motor function recovery between rehabilitation training and resveratrol treatment.

In conclusion, rehabilitation training and resveratrol can individually and synergistically improve the recovery of neurological function and motor function in rats after cerebral ischemic injury and this therapeutic effect is likely achieved through the upregulation of the BDNF/TrkB signaling pathway.

\section{Competing Interests}

All authors certify that they have no affiliations with or involvement in any organization or entity with any financial interest (such as honoraria; educational grants; participation 
in speakers' bureaus; membership, employment, consultancies, stock ownership, or other equity interests; and expert testimony or patent-licensing arrangements) or nonfinancial interest (such as personal or professional relationships, affiliations, knowledge, or beliefs) in the subject matter or materials discussed in this manuscript.

\section{References}

[1] G. Liu, "Research progress of ischemic cerebrovascular diseases," Practical Journal of Cardiac Cerebral Pneumal and Vascular Disease, vol. 21, pp. 5-6, 2013.

[2] Y. Suzuki, N. Nagai, and K. Umemura, "A review of the mechanisms of blood-brain barrier permeability by tissue-type plasminogen activator treatment for cerebral ischemia," Frontiers in Cellular Neuroscience, vol. 10, article 2, 2016.

[3] E. Behravan, B. M. Razavi, and H. Hosseinzadeh, "Review of plants and their constituents in the therapy of cerebral ischemia," Phytotherapy Research, vol. 28, no. 9, pp. 1265-1274, 2014.

[4] Y. Zhang, C. Li, and L. Niu, "Effects of rehabilitation training on motor function recovery and cAMP-PKA signal transduction pathway after ischemic stroke in rats," Acta Laboratorium Animalis Scientia Sinica, vol. 21, pp. 46-49, 2013.

[5] L. Niu, Y. Zhang, C. Li, B. Liu, Y. LJiang, and L. Li, “cAMP /PKA-pCREB signal transduction pathway may mediate a promoting effect of rehabilitation training on motor function after ischemic stroke in rats," Acta Laboratorium Animalis Scientia Sinica, vol. 22, pp. 24-29, 2014.

[6] T. Gan, L. Li, and S. Chen, "Effect of rehabilitation training on the recovery of motor function and expression of Nogo-A and $\mathrm{Ng} \mathrm{R}$ in rats with focal cerebral ischemia reperfusion injury," Chinese Journal of Rehabilitation Medicine, vol. 28, pp. 768-770, 2013.

[7] T. L. Briones, J. Woods, M. Wadowska, M. Rogozinska, and M. Nguyen, "Astrocytic changes in the hippocampus and functional recovery after cerebral ischemia are facilitated by rehabilitation training," Behavioural Brain Research, vol. 171, no. 1, pp. $17-25,2006$.

[8] J.-S. Seo, M.-H. Moon, J.-K. Jeong et al., "SIRT1, a histone deacetylase, regulates prion protein-induced neuronal cell death," Neurobiology of Aging, vol. 33, no. 6, pp. 1110-1120, 2012.

[9] G. Zu, A. Ji, T. Zhou, and N. Che, "Clinicopathological significance of SIRT1 expression in colorectal cancer: a systematic review and meta analysis," International Journal of Surgery, vol. 26, pp. 32-37, 2016.

[10] Y. Wang, Y. Liang, and P. M. Vanhoutte, "SIRT1 and AMPK in regulating mammalian senescence: a critical review and a working model," FEBS Letters, vol. 585, no. 7, pp. 986-994, 2011.

[11] F. Xue, J. W. Huang, P. Y. Ding et al., "Nrf2/antioxidant defense pathway is involved in the neuroprotective effects of Sirtl against focal cerebral ischemia in rats after hyperbaric oxygen preconditioning," Behavioural Brain Research, vol. 309, pp. 1-8, 2016.

[12] K. B. Koronowski and M. A. Perez-Pinzon, "Sirtl in cerebral ischemia," Brain Circulation, vol. 1, no. 1, pp. 69-78, 2015.

[13] Z. Meng, J. Li, H. Zhao et al., "Resveratrol relieves ischemiainduced oxidative stress in the hippocampus by activating SIRT1," Experimental and Therapeutic Medicine, vol. 10, no. 2, pp. 525-530, 2015.

[14] Y. Hattori, Y. Okamoto, K. Nagatsuka et al., "SIRT1 attenuates severe ischemic damage by preserving cerebral blood flow," NeuroReport, vol. 26, no. 3, pp. 113-117, 2015.
[15] Y. Yang, W. Duan, Y. Li et al., "New role of silent information regulator 1 in cerebral ischemia," Neurobiology of Aging, vol. 34, no. 12, pp. 2879-2888, 2013.

[16] Z. Ungvari, Z. Bagi, A. Feher et al., "Resveratrol confers endothelial protection via activation of the antioxidant transcription factor Nrf2," American Journal of Physiology-Heart and Circulatory Physiology, vol. 299, no. 1, pp. H18-H24, 2010.

[17] L.-G. Huang, J.-P. Li, X.-M. Pang et al., "MicroRNA-29c correlates with neuroprotection induced by FNS by targeting both Birc2 and Bak1 in rat brain after stroke," CNS Neuroscience and Therapeutics, vol. 21, no. 6, pp. 496-503, 2015.

[18] K. J. Livak and T. D. Schmittgen, "Analysis of relative gene expression data using real-time quantitative PCR and the $2^{-\Delta \Delta C_{T}}$ method," Methods, vol. 25, no. 4, pp. 402-408, 2001.

[19] J.-P. Wang, C. Liu, Z.-T. Yang, C. Jiang, Y.-Z. Zhao, and D.-Q. $\mathrm{Xu}$, "Effects of resveratrol on brain derived neurotrophic factor and neurotrophin-3 of cerebral ischemia of mice," Chinese Pharmacological Bulletin, vol. 28, no. 4, pp. 531-535, 2012.

[20] J. Zhao, H. Xu, Y. Tian, M. Hu, and H. Xiao, "Effect of electroacupuncture on brain-derived neurotrophic factor mRNA expression in mouse hippocampus following cerebral ischemiareperfusion injury," Journal of Traditional Chinese Medicine, vol. 33, no. 2, pp. 253-257, 2013.

[21] X. L. Shu, Y. S. Zhang, H. Xu, K. Kang, and D. L. Cai, "Brainderived neurotrophic factor inhibits glucose intolerance after cerebral ischemia," Neural Regeneration Research, vol. 8, no. 25, pp. 2370-2378, 2013.

[22] C. D. Pandya, A. Kutiyanawalla, and A. Pillai, "BDNF-TrkB signaling and neuroprotection in schizophrenia," Asian Journal of Psychiatry, vol. 6, no. 1, pp. 22-28, 2013.

[23] R. Andero, D. C. Choi, and K. J. Ressler, "BDNF-TrkB receptor regulation of distributed adult neural plasticity, memory formation, and psychiatric disorders," Progress in Molecular Biology and Translational Science, vol. 122, pp. 169-192, 2014.

[24] R.-Q. Yao, D.-S. Qi, H.-L. Yu, J. Liu, L.-H. Yang, and X.-X. Wu, "Quercetin attenuates cell apoptosis in focal cerebral ischemia rat brain via activation of BDNF-TrkB-PI3K/Akt signaling pathway," Neurochemical Research, vol. 37, no. 12, pp. 2777-2786, 2012.

[25] B. Qiu, S. Hu, L. Liu et al., "CART attenuates endoplasmic reticulum stress response induced by cerebral ischemia and reperfusion through upregulating BDNF synthesis and secretion," Biochemical and Biophysical Research Communications, vol. 436, no. 4, pp. 655-659, 2013.

[26] J.-Y. Chung, M.-W. Kim, M.-S. Bang, and M. Kim, "Increased expression of neurotrophin 4 following focal cerebral ischemia in adult rat brain with treadmill exercise," PLOS ONE, vol. 8, no. 3, Article ID e52461, 2013.

[27] R. Bai, X. Liang, and K. Wang, "Effects of rehabilitation training on the neurological behavior and the expression of BDNF, CaBP-D28k for focal cerebral ischemia of rats," Shangdong Medicine Journal, vol. 52, 2012.

[28] F. Angelucci, J. Piermaria, F. Gelfo et al., “The effects of motor rehabilitation training on clinical symptoms and serum BDNF levels in Parkinson's disease subjects," Canadian Journal of Physiology and Pharmacology, vol. 94, no. 4, pp. 455-461, 2016.

[29] Y. Zeng and K. Yang, "Sirtuin 1 participates in the process of age-related retinal degeneration," Biochemical and Biophysical Research Communications, vol. 468, no. 1-2, pp. 167-172, 2015.

[30] M. Jiang, J. Wang, J. Fu et al., "Neuroprotective role of Sirtl in mammalian models of Huntington's disease through activation 
of multiple Sirtl targets," Nature Medicine, vol. 18, no. 1, pp. 153158, 2012.

[31] G. Wang, B. Liu, J. Lv, Y. Zhao, and R. Zhao, "The expressions of brain-derived neurotrophic factor and tyrosine kinase B in the rats model of ischemic postconditioning," Journal of Brain and Nervous Diseases, vol. 24, pp. 34-39, 2016. 


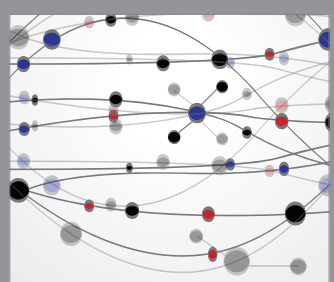

The Scientific World Journal
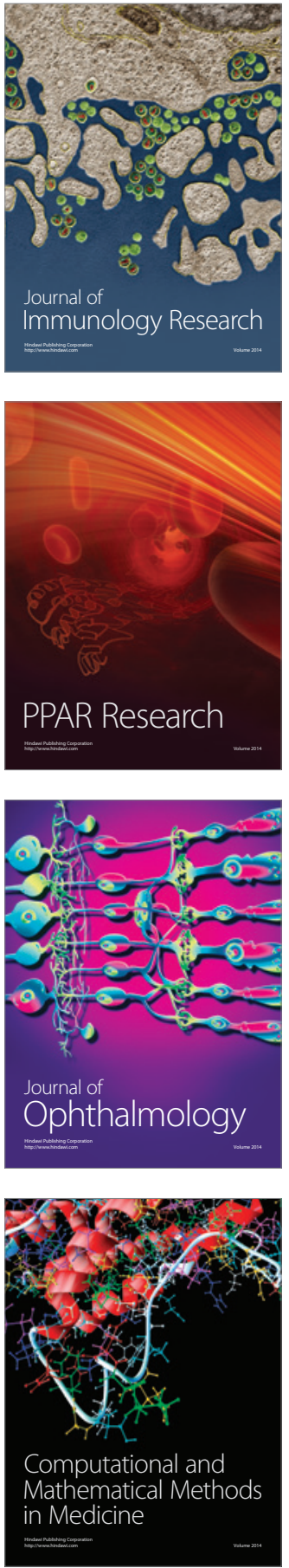

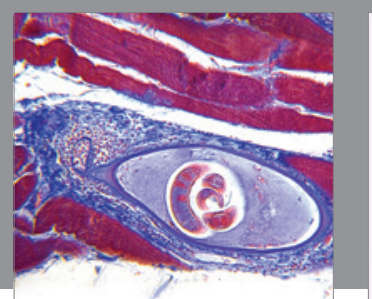

Gastroenterology Research and Practice

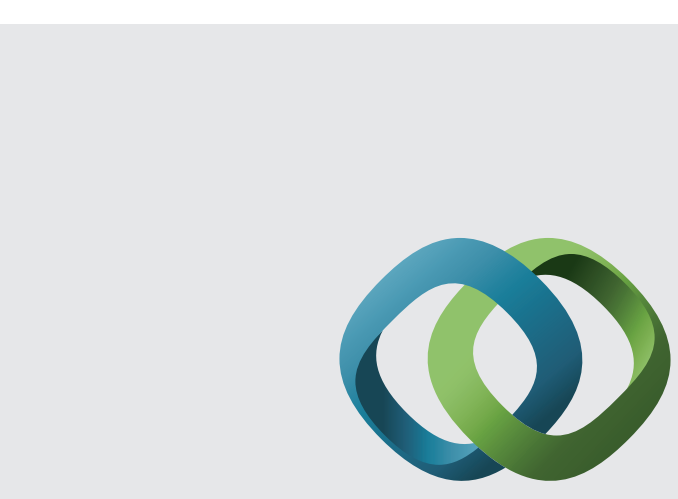

\section{Hindawi}

Submit your manuscripts at

http://www.hindawi.com
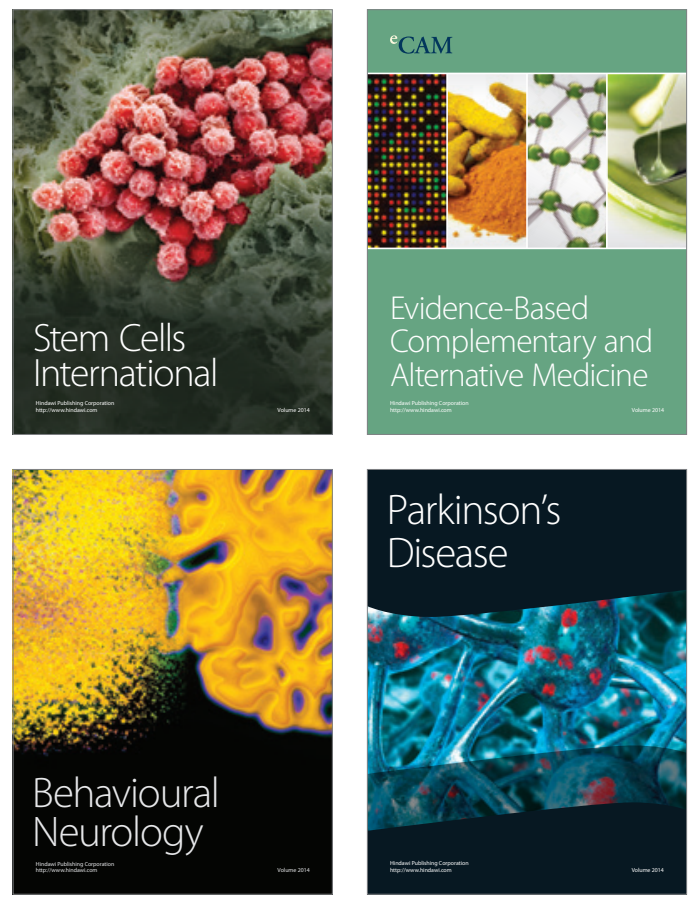
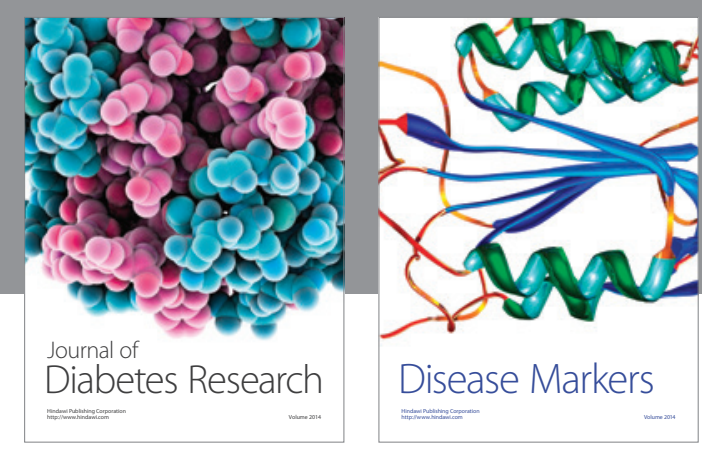

Disease Markers
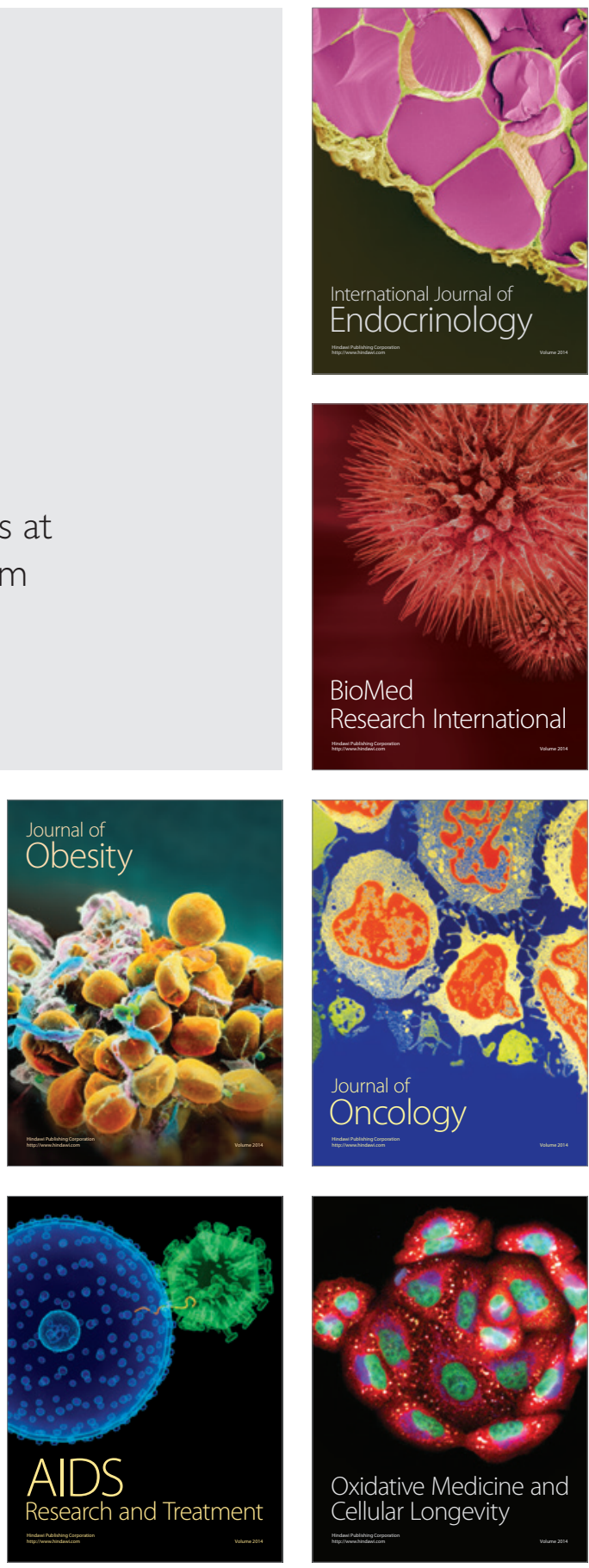